\title{
THE LAMINAR FREE-CONVEGTION BOUNDARY LAYER ON A VERTIGAL HEATED PLATE IN THE NEIGHBOURHOOD OF A DISCONTINUITY IN PLATE TEMPERATURE
}

\author{
R. K. SMITH ${ }^{1}$ \\ (Received 2 January 1969: revised 10 February 1969) \\ Communicated by A. F. Pillow
}

\section{Introduction}

If velocity and temperature profiles are known at a particular distance along a vertical heated plate, the equations of motion determine the velocity and temperature at points downstream, for a given variation of plate temperature. The problem of continuing the boundary layer solution for given initial conditions was investigated by Goldstein [2], for the isothermal case of the laminar, incompressible flow past a flat plate, with a given streamwise variation of pressure gradient outside the boundary layer. He showed that the solution is not always free from singularities and developed an expansion procedure to calculate the flow downstream when these occurred. Typical singularities occur, for instance, near the leading edge of the plate where the no-slip condition is imposed on the plate surface and near the trailing edge, where this condition is relaxed to one of zero stress along the axis of symmetry of the wake.

The method of Goldstein has been applied by Rheinboldt [6] and Watson [8] to the flow with suction or blowing of the boundary layer. It is extended here to advance the solution for the free convection boundary layer along a vertical heated plate, past the height at which a discontinuity of plate temperature occurs. It is assumed that the plate is flat and is maintained at constant temperatures $T_{1}, T_{2}$, below and above a height $L$, above its lower edge. The environment is assumed to be at rest at uniform temperature $T_{0}$ and the Prandtl number of the fluid is taken to be unity ${ }^{2}$. Results are obtained for the two subcases, $T_{2}>T_{1}>T_{0}$ and $T_{1}>T_{2}>T_{0}$.

1 Present address: Department of Mathematics, Monash University, Clayton, Victoria 3168, Australia.

2 The theory can be applied to fluids with Prandtl numbers of order unity but not to those with Prandtl numbers large or small compared to one--see $\$ 7$. 
A pronounced, secondary thermal layer is formed at the temperature discontinuity and grows downstream along the plate. The velocity field responds more gradually to the discontinuity since the velocity conditions on the plate are unaltered. Hence, the subsequent motions of fluid particles near the plate are determined by the buoyancy field of the new thermal layer, which establishes itself downstream at a steady rate. The solutions emphasize the speed with which the temperature field adjusts itself to the abrupt change in plate temperature.

\section{The equations of motion}

The laminar free-convection flow about a vertical, uniformly heated plate has been studied by a number of authors (see Ostrach [5] for references). For a rigorous derivation of the equations, the reader is referred to Ostrach [4].

Let $L$ be a representative length along the plate; $T_{0}$ and $T_{1}$ the ambient and plate temperature and $\beta, v, \kappa$, the volumetric coefficients of expansion, kinematic viscosity and thermometric conductivity of the fluid. The flow is characterised by two dimensionless parameters; the Grashof number,

$$
\mathrm{Gr}=g \beta\left(T_{1}-T_{0}\right) L^{3} / v^{2},
$$

comparing buoyancy to viscous terms in the equations, and the Prandtl number $\sigma=v / \kappa$, comparing the molecular diffusivity of momentum to that of heat. Free-convection flows occur for a range of Gr much larger than unity.

The equations of motion in non-dimensional form are,

$$
\begin{gathered}
\frac{\partial u}{\partial x}+\frac{\partial v}{\partial y}=0, \\
u \frac{\partial u}{\partial x}+v \frac{\partial u}{\partial y}=\theta+\frac{\partial^{2} u}{\partial y^{2}}, \\
u \frac{\partial \theta}{\partial x}+v \frac{\partial \theta}{\partial y}=\frac{1}{\sigma} \frac{\partial^{2} \theta}{\partial y^{2}},
\end{gathered}
$$

where $x, y$ are co-ordinates measured along and perpendicular to the plate from the leading edge; $u, v$, are the velocity components in these directions and $\theta$ is a scaled temperature difference given by $\theta=\left(T-T_{0}\right) /\left(T_{1}-T_{0}\right)$, where $T$ is the temperature at a general position in the flow. (Here, scales $L,\left(v^{2} L / \bar{\theta}\right) \frac{1}{t},(L \bar{\theta})^{\frac{1}{2}},\left(v^{2} \bar{\theta} / L\right)^{\frac{1}{2}}$, have been taken, corresponding to the quantities $x, y, u, v$, where $\bar{\theta}=g \beta\left(T_{1}-T_{0}\right)$, is a scale for the buoyant acceleration term.) 
For a plate maintained at a uniform temperature in an otherwise still environment, the full boundary conditions are:

$$
\begin{array}{ll}
u=\theta=0 & \text { on } \quad x=0, y>0 \\
u=v=0, \theta=1 & \text { on } \quad y=0, x>0 \\
u, \theta \rightarrow 0 & \text { as } \quad y \rightarrow \infty, x>0
\end{array}
$$

Equations $(2.1)-(2.3)$ are reduced by introducing a stream function $\psi$, defined by the relations

$$
u=\psi_{v}, \quad v=-\psi_{x},
$$

and taking

$$
\begin{aligned}
& \psi=x F(\zeta), \\
& \theta=G(\zeta),
\end{aligned}
$$

where

$$
\zeta=y x^{-1}
$$

In terms of $\psi$ equation (2.1) is satisfied identically and equations (2.2) and (2.3) reduce to two ordinary differential equations for $F$ and $G$, thus

$$
\begin{aligned}
& F^{\prime \prime \prime}+\frac{3}{4} F F^{\prime \prime}-\frac{1}{2} F^{\prime 2}+G=0, \\
& G^{\prime \prime}+\frac{3}{4} \sigma F G^{\prime}=0 .
\end{aligned}
$$

Also, from (2.5), the velocity components are given by

$$
\begin{aligned}
& u=x^{\frac{1}{2}} F^{\prime}, \\
& v=\frac{1}{2} x^{-\frac{1}{2}}\left(\zeta F^{\prime}-3 F\right) .
\end{aligned}
$$

Equations (2.8) and (2.9) for $F$ and $G$ have been solved numerically subject to conditions (2.4), for a wide range of Prandtl numbers and details are given in Ostrach [4]. The computations for $\sigma=1$ were repeated here using a routine facility for two-point boundary value problems of this type on the Manchester Atlas Computer. In this case, $F^{\prime \prime}(0)=.90797$ and $G^{\prime}(0)=-.40103$.

\section{The continuation problem}

The continuation problem outlined in $\S 1$ is to advance the solution to equations $(2.1)-(2.3)$, given velocity and temperature profiles at $x=0$ and the variation of temperature along the plate; i.e. 


$$
\begin{aligned}
& u=u_{0}(y), \quad \theta=\theta_{0}(y) \quad \text { on } x=0, y>0 \text {; } \\
& \left.\begin{array}{l}
u=v=0 \quad(x \geqq 0) \\
\theta=\Theta_{0}=\Theta_{1} x+\Theta_{2} x^{2}+\cdots(x>0),
\end{array}\right\} \text { on } y=0 ; \\
& u, \theta \rightarrow 0 \text { as } y \rightarrow \infty, x>0 \text {; }
\end{aligned}
$$

where

and

$$
u_{0}(y)=a_{1} y+a_{2} y^{2}+a_{3} y^{3}+\cdots\left(a_{1} \neq 0\right),
$$

$$
\theta_{0}(y)=b_{0}+b_{1} y+b_{2} y^{2}+\cdots
$$

near $y=0$.

(Note: We restrict ourselves here to initial velocity profiles with a single zero at the origin; if $u$ is finite at the origin, the problem becomes one of mixed or forced convection; if $a_{1}=0$, severe complications arise and the equations are unmanageable - see Goldstein op. cit.).

If there is no singularity at $x=0$, we can expand $\psi$ and $\theta$ as double power series in $x$ and $y$. If these series are substituted into equations (2.1) $-(2.3)$ and the boundary conditions (3.1) are satisfied, we find that certain relations must hold between the coefficients $a_{i}, b_{i}$ and $\Theta_{i}$ in (3.1). The first few are

$$
\begin{array}{lll}
b_{0}+2 ! a_{2}=0, & b_{1}+3 ! a_{3}=0, & 18 b_{3}+5 ! a_{5}=0, \cdots, \\
b_{0}=\Theta_{0}, & b_{2}=0, & 3 ! b_{3}=a_{1} \Theta_{1}, \cdots .
\end{array}
$$

Further, if these conditions hold, we have

$$
\left(\frac{\partial \theta}{\partial y}\right)_{y=0}=a_{1}+\frac{4 ! a_{4}}{a_{1}} x+\cdots
$$

and

$$
\left(\frac{\partial \theta}{\partial y}\right)_{y=0}=b_{1}+\frac{4 !\left(a_{1} b_{4}-a_{4} b_{1}\right)+a_{1} \Theta_{0} \Theta_{1}}{2 a_{1}^{2}} x+\cdots
$$

The coefficient $a_{1}, a_{4}, b_{1}, b_{4}$, etc. are not determined by the relations (3.2); once these are specified, we can find the skin friction and local beat transfer from the plate, which are proportional to (3.3) and (3.4) respectively.

If the relations (3.2) are not satisfied, there is an algebraic singularity at $x=0$.

\section{Inner expansion}

With the stream function $\psi$ defined by equation (2.5), we make the following transformation of variables 


$$
\xi=x^{\frac{1}{3}}, \quad \eta=\frac{1}{3} y x^{-\frac{1}{3}},
$$

and take

$$
\psi=\xi^{2} f(\xi, \eta)
$$

and

$$
\theta=\frac{1}{27} \xi^{-1} g(\xi, \eta)
$$

Then, from (2.5) and (4.2), the velocity components are given by

$$
\begin{aligned}
& u=\frac{1}{3} \xi f_{\eta} \\
& v=-\frac{1}{3} \xi^{-1}\left[2 f+\xi f_{\xi}-\eta f_{\eta}\right] .
\end{aligned}
$$

Further, equations (2.2) and (2.3) become

$$
\begin{aligned}
f_{\eta}^{2}+\xi f_{\eta \xi} f_{\eta}-2 f f_{\eta}-\xi f_{\xi} f_{\eta \eta} & =f_{\eta \eta \eta}+g, \\
-f_{\eta} g+\xi g_{\xi} f_{\eta}-2 f g_{\eta}-\xi f_{\xi} g_{\eta} & =\frac{1}{\sigma} g_{\eta \eta} .
\end{aligned}
$$

The transformed boundary conditions from (3.1) are

$$
\begin{aligned}
& \left.\begin{array}{ll}
f=f_{\eta}=0, & (\xi \geqq 0), \\
g=27 \xi\left[\Theta_{0}+\Theta_{1} \xi^{3}+\Theta_{2} \xi^{6}+\cdots\right) & (\xi>0),
\end{array}\right\} \text { on } \eta=0, \\
& \left.\begin{array}{l}
f_{\eta}=3 \xi^{-1}\left(a_{1}(3 \xi \eta)+a_{2}(3 \xi \eta)^{2}+\cdots\right), \\
g=27 \xi\left(b_{0}+b_{1}(3 \xi \eta)+\cdots\right.
\end{array}\right\} \text { as } \xi \rightarrow 0 \text { and } \eta \rightarrow \infty .
\end{aligned}
$$

Guided by this form, we expand $f$ and $g$ as power series in $\xi$. Thus

$$
\begin{aligned}
& f=f_{0}+\xi f_{1}+\xi^{2} f_{2}+\cdots, \\
& g=\xi_{1}+\xi^{2} g_{2}+\xi^{3} g_{3}+\cdots
\end{aligned}
$$

where $f_{r}$ and $g_{r}$ are functions of $\eta$ only. Inserting (4.8) and (4.9) into (4.5) and (4.6), we obtain two sets of ordinary differential equations for $f_{r}$ and $g_{r}$. These are:

and

$$
\begin{aligned}
& f_{0}^{\prime \prime \prime}+2 f_{0} f_{0}^{\prime \prime}-f_{0}^{\prime 2}=0, \\
& f_{1}^{\prime \prime \prime}+2 f_{0} f_{1}^{\prime \prime}-3 f_{0}^{\prime} f_{1}^{\prime}+3 f_{0}^{\prime \prime} f_{1}=-g_{1}, \\
& f_{2}^{\prime \prime \prime}+2 f_{0} f_{2}^{\prime \prime}-4 f_{0}^{\prime} f_{2}^{\prime}+4 f_{0}^{\prime \prime} f_{2}=-g_{2}-3 f_{1} f_{1}^{\prime \prime}+2 f_{1}^{\prime 2}, \\
& f_{3}^{\prime \prime \prime}+2 f_{0} f_{3}^{\prime \prime}-5 f_{0}^{\prime} f_{3}^{\prime}+5 f_{0}^{\prime \prime} f_{3}=-g_{3}-4 f_{2} f_{1}^{\prime \prime}+5 f_{2}^{\prime} f_{1}^{\prime}-3 f_{2}^{\prime \prime} f_{1},
\end{aligned}
$$

$$
\begin{aligned}
& g_{1}^{\prime \prime}+2 \sigma f_{0} g_{1}^{\prime}=0, \\
& g_{2}^{\prime \prime}+2 \sigma f_{0} g_{2}^{\prime}-\sigma f_{0}^{\prime} g_{2}=-3 \sigma f_{1} g_{1}^{\prime},
\end{aligned}
$$$$
g_{3}^{\prime \prime}+2 \sigma f_{0} g_{3}^{\prime}-2 \sigma f_{0}^{\prime} g_{3}=\sigma\left(f_{1}^{\prime} g_{2}-3 f_{1} g_{2}^{\prime}-4 f_{2} g_{1}^{\prime}\right) \text {, }
$$$$
g_{4}^{\prime \prime}+2 \sigma f_{0} g_{4}^{\prime}-3 \sigma f_{0}^{\prime} g_{4}=\sigma\left(2 f_{1}^{\prime} g_{3}+f_{2}^{\prime} g_{2}-3 f_{1} g_{3}^{\prime}-4 f_{2} g_{2}^{\prime}-5 f_{3} g_{1}^{\prime}\right) \text {. }
$$ 
Comparing conditions (4.7) with the series (4.8) and (4.9), we obtain the following boundary conditions for $f_{r}$ and $g_{r}$,

$$
\begin{array}{ll}
f_{r}=f_{r}^{\prime}=0 & \text { or } \eta=0, \\
g_{3 r+1}=27 \Theta_{r}, \quad g_{3 r}=g_{3 r+2}=0 & \text { or } \eta=0, \\
\lim _{\eta \rightarrow \infty} \frac{f_{r}^{\prime}(\eta)}{\eta^{r+1}}=3^{r+2} a_{r+1}, & \\
\lim _{\eta \rightarrow \infty} \frac{g_{r-1}(\eta)}{\eta^{r}}=3^{r+3} b_{r} . &
\end{array}
$$

The solution for $f_{0}$ having a double zelo at the origin and satisfying (4.13) is

$$
f_{0}=\frac{9}{2} a_{1} \eta^{2}
$$

If we take

$$
z=\alpha \eta=\left(9 a_{1}\right)^{\frac{1}{\eta}} \eta,
$$

then

$$
f_{0}=\frac{1}{2} \alpha z^{2}=A_{0} z^{2},
$$

and these last two equations define $\alpha$ and $A_{0}$.

The equation for $g_{1}$ is now

$$
\frac{d^{2} g_{1}}{d z^{2}}+\sigma z^{2} \frac{d g_{1}}{d z}=0 .
$$

This equation can be integrated directly and the solution satisfying conditions (4.12b) and (4.14) is

$$
g_{1}=\frac{27\left(b_{0}-\Theta_{0}\right)}{\Gamma\left(\frac{1}{3}\right)} \gamma\left(\frac{1}{3}, \frac{1}{3} \sigma z^{3}\right)+27 \Theta_{0}
$$

where

$$
\gamma(n, x)=\int_{0}^{x} t^{n-1} e^{-t} d t,
$$

is the incomplete gamma function and

$$
\Gamma(n)-\gamma(n, x) \sim e^{-x} x^{n-1}\left(1+\frac{n-1}{x}+\frac{(n-1)(n-2)}{x^{2}}+\ldots\right) \text { as } x \rightarrow \infty .
$$

We note in passing that

$$
g_{1}^{\prime}=\frac{27\left(b_{0}-\Theta_{0}\right) \sigma^{3} 3^{2}}{\Gamma\left(\frac{1}{3}\right)} e^{-\frac{1}{3} \sigma z^{3}} .
$$


In terms of $z$, the equations for $f_{r}$ and $g_{r}$ can be written

$$
\frac{d^{3} f_{r}}{d z^{3}}+z^{2} \frac{d^{2} f_{r}}{d z^{2}}-(r+2) z \frac{d f_{r}}{d z}+(r+z) f_{r}=-\frac{g_{r}}{9 a_{1}}+\alpha^{-1} F_{r}
$$

and

$$
\frac{d^{2} g_{r}}{d z^{2}}+\sigma z^{2} \frac{d g_{r}}{d z}-\sigma(r-1) z g_{r}=\alpha^{-1} G_{r}
$$

where $F_{r}$ and $G_{r}$ are functions involving $f_{i}, g_{i}(1 \leqq i \leqq r-1)$ and their derivatives.

Complementary functions for $f_{r}$, satisfying equation (4.21) with zero right hand side, are obtained by a power series substitution

$$
f_{r}=\sum_{n=0}^{\infty} c_{n} z^{n}
$$

The recurrence relation, obtained by equating powers of $z$, is

$$
c_{n+3}=-\frac{(n-1)(n-r-2)}{(n+3)(n+2)(n+1)} c_{n} .
$$

The solutions can be conveniently expressed as generalized hypergeometric functions of the type ${ }_{2} F_{2}$ and three independent complementary functions $f_{r i}(i=1,2,3)$, are:

$$
\begin{aligned}
& t_{r 1}={ }_{2} F_{2}\left(-\frac{1}{3},-\frac{1}{3} r-\frac{2}{3} ; \frac{1}{3}, \frac{2}{3} ;-\frac{1}{3} z^{3}\right), \\
& f_{r 2}=z, \\
& t_{r 3}=z^{2}{ }_{2} F_{2}\left(\frac{1}{3},-\frac{1}{3} r ; \frac{5}{3}, \frac{4}{3} ;-\frac{1}{3} z^{3}\right) .
\end{aligned}
$$

Complementary functions for $g_{r}(r>1)$ are found as follows. The substitution $s=z^{3}$ in equation (4.22) with zero right hand side gives

Inserting

$$
9 s \frac{d^{2} g_{r}}{d s^{2}}+3(2+\sigma s) \frac{d g_{r}}{d s}-\sigma(r-1) g_{r}=0 .
$$

$$
g_{r}=\sum_{n=0}^{\infty} c_{n} s^{\rho+n}
$$

into this equation and equating powers of $s$, we obtain the recurrence relation

$$
c_{n+1}=\frac{-\frac{1}{3} \sigma\left(n+\rho-\frac{r+1}{3}\right)}{(n+\rho+1)(n+\rho+2)} c_{n} .
$$

The coefficient of $z^{0}$ gives the indicial equation

$$
3 \rho(3 \rho-1)=0,
$$


from which $\rho=0$, and $\rho=\frac{1}{3}$, give independent solutions. These solutions, $g_{r i}(i=1,2)$ say, can be expressed in terms of the confluent hypergeometric function. Thus, in terms of $z$,

$$
\begin{aligned}
& g_{r 1}={ }_{1} F_{1}\left(-\frac{r-1}{3} ; \frac{2}{3} ;-\frac{1}{3} \sigma z^{3}\right), \\
& g_{r 2}=z_{1} F_{1}\left(-\frac{r-2}{3} ; \frac{4}{3} ;-\frac{1}{3} \sigma z^{3}\right) .
\end{aligned}
$$

The boundary conditions on $f_{r}$ and $g_{r}$ as $z \rightarrow \infty$, are

$$
\lim _{z \rightarrow \infty} \frac{f_{r}^{\prime}(z)}{z^{r+1}}=\left(\frac{3}{\alpha}\right)^{r+2} a_{r+1}
$$

and

$$
\lim _{z \rightarrow \infty} \frac{g_{r+1}(z)}{z^{r}}=\frac{3^{r+3}}{\alpha^{r}} b_{r} .
$$

To apply these conditions, we must investigate the asymptotic behaviour of $f_{r}$ and $g_{r}$, and thus the behaviour of the functions $f_{r i}$ and $g_{r i}$, for large $z$.

Asymptotic series for $f_{r 1}$ and $f_{r 3}$ have been obtained by Goldstein op. cit.. These are quoted below.

If $r=3 n$ for some integer $n, f_{r 1}$ is a finite series. Similarly for $f_{r 3}$ if $r=3 n+1 ; g_{r 1}$ if $r=3 n+1 ; g_{r^{2}}$ if $r=3 n-1$.

If $r \neq 3 n-1$,

$$
\begin{aligned}
f_{r 1} \sim- & \frac{z^{r+2} \Gamma\left(\frac{1}{\underline{3}}\right)}{3^{(r+2) / 3} \Gamma\left(\frac{r+4}{3}\right)} \sum_{\mu=0}^{N} \frac{\left(-\frac{r}{3}\right)_{\mu}\left(-\frac{r+2}{3}\right)_{\mu}}{(r+1-3 \mu) \mu !}\left(\frac{1}{3} z^{3}\right)^{-\mu} \\
+ & \frac{\Gamma\left(\frac{1}{3}\right) \Gamma\left(-\frac{r+1}{3}\right)_{z}}{3^{\frac{1}{3}} \Gamma\left(-\frac{r+2}{3}\right)}+O\left(z^{-r=3 N-1}\right)
\end{aligned}
$$

and

$$
\begin{gathered}
f_{r 3} \sim \frac{z^{r+2} \Gamma\left(\frac{5}{3}\right)}{3^{r / 3} \Gamma\left(\frac{r+5}{3}\right)} \sum_{\mu=0}^{N} \frac{\left(-\frac{r}{3}\right)_{\mu}\left(-\frac{r+2}{3}\right)_{\mu}}{(r+1-3 \mu) \mu !}\left(\frac{1}{3} z^{3}\right)^{-\mu} \\
+\frac{3^{\frac{1}{3}} \Gamma\left(\frac{5}{3}\right) \Gamma\left(-\frac{r+1}{3}\right)_{z}}{\Gamma\left(-\frac{r}{3}\right)}+O\left(z^{-r-3 N-1}\right) .
\end{gathered}
$$


If $r=3 n-1$, the terms with $\mu=n$, in the $\sum$-expression of each series must be omitted and the last term in (4.27) replaced by

$$
\frac{(-1)^{n} \Gamma\left(\frac{1}{3}\right) z}{3^{\frac{1}{3}} \Gamma(n+1) \Gamma\left(-\frac{1}{3}-n\right)}\left[\log \left(\frac{1}{3} z^{3}\right)+\psi(n+1)-\psi\left(\frac{2}{3}\right)-\psi\left(-\frac{1}{3}\right)\right],
$$

and the last term in (4.28) replaced by

$$
\frac{(-1)^{r} 3^{\frac{1}{3}} \Gamma\left(\frac{5}{3}\right) z}{\Gamma(n+1) \Gamma\left(\frac{1}{3}-n\right)}\left[\log \left(\frac{1}{3} z^{3}\right)+\psi(n+1)-\psi\left(\frac{4}{3}\right)-\psi\left(\frac{1}{3}\right)\right],
$$

where $\psi(z)^{3}$ is the logarithmic derivative of $\Gamma(z)$.

The asymptotic expansion of the confluent hypergeometric function with negative argument is given by

$$
{ }_{1} F_{1}(a ; b ;-s) \sim \frac{\Gamma(b)}{\Gamma(b-a)} \sum_{\mu=0}^{N} \frac{(a)_{\mu}(1+a-b)_{\mu}}{\mu !} s^{-a-\mu}+O\left(s^{-a-N-1}\right),
$$

(see Slater, 1964). Thus, the expansions for $g_{r i}$ are

$$
g_{r 1} \sim z^{r-1}\left(\frac{\sigma}{3}\right)^{(r-1) / 3} \frac{\Gamma\left(\frac{2}{3}\right)}{\Gamma\left(\frac{r+1}{3}\right)} \sum_{\mu=0}^{N} \frac{\left(-\frac{r-1}{3}\right)_{\mu}\left(-\frac{r-2}{3}\right)_{\mu}}{\mu !}\left(\frac{1}{3} \sigma z^{3}\right)^{-\mu},
$$

and

$$
g_{r 2} \sim z^{r-1}\left(\frac{\sigma}{3}\right)^{(r-2) / 3} \frac{\Gamma\left(\frac{4}{3}\right)}{\Gamma\left(\frac{r+2}{3}\right)} \sum_{\mu=0}^{N} \frac{\left(-\frac{r-1}{3}\right)_{\mu}\left(-\frac{r-2}{3}\right)_{\mu}}{\mu !}\left(\frac{1}{3} \sigma z^{3}\right)^{-\mu}
$$

The solution for $f_{1}$. The equation for $f_{1}$ is

$$
\frac{d^{3} f_{1}}{d z^{3}}+z^{2} \frac{d^{2} f_{1}}{d z^{2}}-3 z \frac{d f_{1}}{d z}+3 f_{1}=-\frac{g_{1}}{9 a_{1}}
$$

For large $z$,

$$
g_{1} \sim 27 b_{0}+O\left(\exp \left(-\frac{1}{3} \sigma z^{3}\right)\right),
$$

and therefore, a particular integral for $f_{1}$, valid for large $z$, is

$$
f_{1 p} \sim-\frac{b_{0}}{2 a_{1}} z^{3}
$$

The asymptotic behaviours of the complementary functions $f_{r i}$, obtained from the series (4.27) and (4.28) are:

3 Only in this sentence does $\psi$ not denote the stream function. 


$$
\begin{aligned}
& f_{11}=1-\frac{1}{2} z^{3}, \\
& f_{12}=z, \\
& f_{13} \sim A z^{3}+B z+C,
\end{aligned}
$$

where $A, B, C(=-2 A)$, are constants determined by equation (4.28) with $r=1$. In particular,

$$
A=2 \Gamma\left(\frac{2}{3}\right) / 3^{\frac{4}{3}} .
$$

The complete solution for $f_{1}$ may be written

$$
f_{1}=f_{1 p}+r_{1} f_{11}+s_{1} f_{12}+t_{1} f_{13}
$$

where $r_{1}, s_{1}, t_{1}$, are constants. Moreover, the asymptotic behaviour of this solution has the form

$$
f_{1} \sim A_{1} z^{3}+B_{1} z+C_{1} .
$$

The solution with a double zero at the origin and satisfying (4.25) is obtained numerically as follows. Equation (4.33) is integrated outwards with starting values $f_{1}(0)=f_{1}^{\prime}(0)=0, f_{1}^{\prime \prime}(0)=1$, and the derivatives calculated for a large value of $z(z=7$, is found to be sufficiently large). This solution is regarded as a particular integral for $f_{\mathbf{1}}$, say $f_{\mathbf{1 q}}$. Then, the general solution with a double zero at the origin is

$$
f_{1}=f_{1 q}+\lambda_{1} f_{13}
$$

for any value of $\lambda_{1}$. Further

$$
\begin{aligned}
f_{1}^{\prime \prime} & =f_{1 q}^{\prime \prime}+\lambda_{1} f_{13}^{\prime \prime} \\
& \sim f_{1 q}^{\prime \prime}+6 \lambda_{1} A z \quad \text { as } \quad z \rightarrow \infty .
\end{aligned}
$$

From equation $(\mathbf{4 . 3 6})^{4}$ we see that $f_{1 q}^{\prime \prime} / z \rightarrow$ a constant as $z \rightarrow \infty$ and using condition (4.25) we find

$$
\lim _{z \rightarrow \infty} \frac{f_{1}^{\prime \prime}}{z}=\frac{6 a_{2}}{a_{1}}=\lim _{z \rightarrow \infty} \frac{f_{1 q}^{\prime \prime}}{z}+6 \lambda_{1} A
$$

The limit $f_{1}^{\prime \prime} / z$ is obtained numerically and $\lambda$, is then deduced from (4.38). Then from (4.37), we have

$$
f_{1}^{\prime \prime}(0)=f_{1 q}^{\prime \prime}(0)+2 \lambda_{1}=1+2 \lambda_{1} .
$$

Equation (4.33) is now solved numerically with this as the appropriate second derivative at the origin. In the asymptotic expansion for this solution, the coefficient $A_{1}$ in $(4.36)$ has been obtained by satisfying the

4 It can be shown that an asymptotic power series, valid in a sector of the complex plane, can be differentiated (Erdélyi, [1]). 
boundary condition as $z \rightarrow \infty$, i.e. $A_{1}=a_{2} / a_{1}$. The corresponding coefficient $B_{1}$ is calculated numerically using the values for $f_{1}^{\prime}$ and $f_{\mathbf{1}}^{\prime \prime}$. Hence

Also

$$
B_{1}=\lim _{z \rightarrow \infty}\left(f_{1}^{\prime}-\frac{1}{2} z f_{1}^{\prime \prime}\right) \text {. }
$$

$$
C_{1}=-2 A_{1} \text {. }
$$

The solution for $g_{2}$. The equation for $g_{2}$ is

$$
\frac{d^{2} g_{2}}{d z^{2}}+\sigma z^{2} \frac{d g_{2}}{d z}-\sigma z g_{2}=-\frac{3 \sigma}{\alpha} f_{1} g_{1} .
$$

The equation has a particular integral $g_{2 p}$, which is exponentially small for large $z$. The complete solution is therefore

$$
g_{2}=g_{2 p}+m g_{21}+n g_{22},
$$

where $m$ and $n$ are constants and for large $z$,

$$
g_{2} \sim\left(m\left(\frac{\sigma}{3}\right)^{\frac{1}{3}} \Gamma\left(\frac{2}{3}\right)+n\right) z .
$$

Equation (4.39) is integrated outwards with starting values $g_{2}(0)=0$, $g_{2}^{\prime}(0)=1$, to give a particular integral $g_{2 q}$. The general solution with a single zero at the origin is then

$$
\begin{aligned}
g_{2} & =g_{2 q}+\mu g_{22} \\
& \sim\left(\alpha_{\alpha}+\mu\right) z \text { as } z \rightarrow \infty,
\end{aligned}
$$

where $\alpha_{q}=\lim _{z \rightarrow \infty} g_{2}^{\prime}$, is calculated numerically and $\mu$ is any constant. Then, using (4.26)

$$
\lim _{z \rightarrow \infty} \frac{g_{2}}{z}=\frac{3^{4} b_{1}}{\alpha}=\alpha_{a}+\mu,
$$

giving $\mu$. The integration is repeated with $g_{2}^{\prime}(0)$ replaced by $1+\mu$ to give the required solution. For this,

$$
g_{2} \sim \alpha_{1} z=\frac{3^{4} b_{1}}{\alpha} z \text { as } z \rightarrow \infty .
$$

The solution for $f_{2}$. The equation for $f_{2}$ is

$$
\frac{d^{3} f_{2}}{d z^{3}}+z^{2} \frac{d^{2} f_{2}}{d z^{2}}-4 z \frac{d f_{2}}{d z}+4 f_{2}=-\frac{g_{2}}{9 a_{1}}+\frac{2 f_{1}^{\prime 2}-3 f_{1} f_{1}^{\prime \prime}}{\alpha} .
$$

With the asymptotic series for $f_{1}$ given by (4.36) and $g_{2}$ given by (4.42), we can find a particular integral $f_{2 p}$ to this equation such that

$$
f_{2 y} \sim a^{\prime} z^{4}+b^{\prime} z^{2}+c^{\prime} z+d^{\prime}, \quad \text { as } z \rightarrow \infty,
$$


where $a^{\prime}, b^{\prime}, c^{\prime}, d^{\prime}$ are constants depending on $A_{1}, B_{1}, C_{1}$ and $\alpha_{1}$. The general solution for $f_{2}$ is

$$
f_{2}=f_{2 p}+r_{2} f_{21}+s_{2} f_{22}+t_{2} f_{23} \text {, }
$$

where $r_{2}, s_{2}, t_{2}$, are constants. Then, using the asymptotic series for the functions $f_{2 i}$ given above, we find that

$$
f_{2} \sim A_{2} z^{4}+B_{2} z^{2}+C_{2}^{\prime} z \log z+C_{2} z+D_{2}+E_{2} z^{-2}+O\left(z^{-5}\right) \quad \text { as } z \rightarrow \infty .
$$

Also, from equation (4.28), we have

$$
f_{23} \sim \frac{3^{\frac{1}{3}} \Gamma\left(\frac{2}{3}\right)}{6} z^{4}+O\left(z^{2}\right) \quad \text { as } \quad z \rightarrow \infty .
$$

Hence, the general solution for $f_{2}$ with a double zero at the origin is

and

$$
f_{2}=f_{2 q}+\lambda_{2} f_{23}
$$

$$
f_{2}^{\prime} \sim 4\left(A_{2 q}+\lambda_{2} \frac{3^{\frac{3}{3}} \Gamma\left(\frac{2}{3}\right)}{6}\right) z^{3}+O(z) \text { as } z \rightarrow \infty,
$$

where $f_{2 q}$ is the particular integral for which $f_{2 q}^{\prime \prime}(0)=1 ; A_{2 q}$ is the leading coefficient in the asymptotic series for this solution and $\lambda_{2}$ is any constant. The value of $A_{2 q}$ and the next three coefficients in the series (4.44) are obtained numerically. This value of $A_{2 q}$ is used together with (4.25) and (4.47) to find $\lambda_{2}$. The appropriate solution for $t_{2}$ and the corresponding coefficients $A_{2}, B_{2}, C_{2}$ and $C_{2}^{\prime}$ are there found by integrating equation (4.43) numerically with $f^{\prime \prime}(0)=1+2 \lambda_{2}$.

A similar procedure can be used for calculating $g_{3}, f_{3}, g_{4}, f_{4}, g_{5}, \cdots$ etc., in that order. However, the labour involved increases rapidly for higher terms. We therefore restrict ourselves to calculating four terms in each of the series for $f$ and $g$. These appear sufficient to enable a reasonable account to be given of the initial development of the inner layer. The asymptotic series obtained for $g_{3}, f_{3}$ and $g_{4}$ are given below

$$
\begin{aligned}
& g_{3} \sim \alpha_{3} z^{2}+\beta_{3}+\gamma_{3} z^{-1}+O\left(z^{-4}\right), \\
& f_{3} \sim A_{3} z^{5}+B_{3} z^{3}+C_{3}^{\prime} z^{2} \log z+C_{3} z^{2}+D_{3} z+E_{3}^{\prime} \log z+E_{3}+O\left(z^{-1}\right), \\
& g_{4} \sim \alpha_{4} z^{3}+\beta_{4}^{\prime} \log z+\beta_{4}+O\left(z^{-1}\right) .
\end{aligned}
$$

\section{Outer expansion}

The series expansions for $\psi$ and $\theta$ obtained in $\S 4$, satisfy the boundary conditions on the plate $(z=0)$ and at the initial section $(x=0)$. However, for large $z$, the expansions have the form 


$$
\begin{aligned}
\psi \sim & A_{0} \xi^{2} z^{2}+\xi^{3}\left(A_{1} z^{3}+B_{1} z+C_{1}\right) \\
& +\xi^{4}\left(A_{2} z^{4}+B_{2} z^{2}+C_{2}^{\prime} z \log z+C_{2} z+D_{2}+E_{2} z^{-2}+\cdots\right) \\
& +\xi^{5}\left(A_{3} z^{5}+B_{3} z^{3}+C_{3}^{\prime} z^{2} \log z+C_{3} z^{2}+D_{3} z+E_{3}^{\prime} \log z+E_{3}+\cdots\right) \\
& +\cdots,
\end{aligned}
$$

and

$$
\begin{array}{r}
\theta \sim \frac{1}{27}\left[\alpha_{1}+\alpha_{2} \xi z+\xi^{2}\left(\alpha_{3} z^{2}+\beta_{3}+\gamma_{3} z^{-1} \xi+\cdots\right)\right. \\
\left.+\xi^{3}\left(\alpha_{4} z^{3}+\beta_{4}^{\prime} \log z+\beta_{4}+\cdots\right)+\cdots\right]
\end{array}
$$

and these both diverge as $z \rightarrow \infty$. The expansions are therefore valid only in a neighbourhood of the plate, in fact for $\xi z \ll 1$. To find a solution valid for large $y x^{-\frac{1}{3}}$, we seek expansions for $\psi$ and $\theta$ in powers of $\xi$, which satisfy the boundary conditions on $x=0$ and as $y \rightarrow \infty$, but not necessarily on $y=0$, and which coincide with the inner solution in some region away from the plate tor each $\xi$.

If it is assumed that rearrangement is possible, the series (5.1) and (5.2) can be written in terms of the outer variable $y$. Then

$$
\begin{aligned}
\psi \sim & A_{0}\left(\frac{1}{3} \alpha y\right)^{2}+A_{1}\left(\frac{1}{3} \alpha y\right)^{3}+A_{2}\left(\frac{1}{3} \alpha y\right)^{4}+\cdots \\
& +\xi^{2}\left(B_{1}\left(\frac{1}{3} \alpha y\right)+B_{2}\left(\frac{1}{3} \alpha y\right)^{2}+B_{3}\left(\frac{1}{3} \alpha y\right)^{3}+\cdots\right) \\
& +\xi^{3}\left(C_{1}+\left(C_{2}+C_{2}^{\prime} \log \left(\frac{1}{3} \alpha\right)\right)\left(\frac{1}{3} \alpha y\right)+C_{2}^{\prime}\left(\frac{1}{3} \alpha y \log y\right)\right. \\
& \left.+\left(C_{3}+C_{3}^{\prime} \log \left(\frac{1}{3} \alpha\right)\right)\left(\frac{1}{3} \alpha y\right)^{2}+C_{3}^{\prime}\left(\frac{1}{3} \alpha y\right)^{2} \log y+\cdots\right) \\
& -\xi^{3} \log \xi\left(C_{2}^{\prime}\left(\frac{1}{3} \alpha y\right)+C_{3}^{\prime}\left(\frac{1}{3} \alpha y\right)^{2}+\cdots\right)+\cdots
\end{aligned}
$$

and

$$
\begin{aligned}
\theta \sim & \frac{1}{27}\left(\alpha_{1}+\alpha_{2}\left(\frac{1}{3} \alpha y\right)+\alpha_{3}\left(\frac{1}{3} \alpha y\right)^{2}+\cdots\right) \\
& +\frac{1}{27} \xi^{2}\left(\beta_{3}+\beta_{4}\left(\frac{1}{3} \alpha y\right)+\cdots\right) \\
& +\frac{1}{27} \xi^{3}\left(\gamma_{3}\left(\frac{1}{3} \alpha y\right)^{-1}+\beta_{4}^{\prime} \log \left(\frac{1}{3} \alpha y\right)+\cdots\right) \\
& -\frac{1}{27} \xi^{3} \log \xi\left(\gamma_{4}+\cdots\right),
\end{aligned}
$$

where $\alpha$ is given by (4.16). We therefore assume that for sufficiently large values of $y x^{-\frac{1}{3}}$, we can write

$$
\psi=\psi_{0}(y)+\frac{\xi^{2}}{2 !} \psi_{2}(y)+\frac{\xi^{3}}{3 !} \psi_{3}(y)+\frac{\xi^{3} \log \xi}{3 !} \bar{\psi}_{3}(y)+O\left(\xi^{4}\right),
$$

and

$$
\theta=\theta_{0}(y)+\frac{\xi^{2}}{2 !} \theta_{2}(y)+\frac{\xi^{3}}{3 !} \theta_{3}(y)+\frac{\xi^{3} \log \xi}{3 !} \theta_{3}(y)+O\left(\xi^{4}\right),
$$

where $\psi_{0}^{\prime}(y)$ and $\theta_{0}(y)$ are given (see 3.1) and near $y=0$, 
and

$$
\begin{aligned}
\psi_{0}^{\prime}= & a_{1} y+a_{2} y^{2}+a_{3} y^{3}+a_{4} y^{4}+\cdots, \\
\psi_{2}= & \frac{2}{3} \alpha B_{1} y+\frac{2}{9} \alpha^{2} B_{1} y^{2}+\frac{2}{27} \alpha^{3} B_{3} y^{3}+\cdots, \\
\psi_{3}= & 6 C_{1}+2 \alpha\left(C_{2}+C_{2}^{\prime} \log \left(\frac{1}{3} \alpha\right)\right) y+2 \alpha C_{2}^{\prime} y \log y \\
& +\frac{2}{3} \alpha^{2}\left(C_{3}+C_{3}^{\prime} \log \left(\frac{1}{3} \alpha\right)\right) y^{2}+\frac{2}{3} \alpha^{2} C_{3}^{\prime} y^{2} \log y+\cdots, \\
\bar{\psi}_{3}= & -2 \alpha C_{2}^{\prime} y-\frac{2}{3} \alpha^{2} C_{3}^{\prime} y^{2}-\cdots ;
\end{aligned}
$$

$$
\begin{aligned}
& \theta_{0}=b_{0}+b_{1} y+b_{2} y^{2}+b_{3} y^{3}+\cdots \\
& \theta_{2}=\frac{2}{27}\left(\beta_{3}+\frac{1}{3} \alpha \beta_{4} y+\cdots\right) \\
& \theta_{3}=\frac{2}{3} \gamma_{3}(\alpha y)^{-1}+\cdots \\
& \bar{\theta}_{3}=\frac{2}{9} \gamma_{4}^{\prime}+\cdots
\end{aligned}
$$

If the series (5.5) and (5.6) are substituted into equations (2.2) and (2.3), making use of (2.5), and the coefficients of $\xi^{r}$ and $\xi^{r} \log \xi$ are equated, the following sets of equations are obtained for the functions $\psi_{i}(y)$ and $\theta_{i}(y)$ :

and

$$
\begin{aligned}
& \psi_{0}^{\prime} \psi_{2}^{\prime}-\psi_{2} \psi_{0}^{\prime \prime}=0, \\
& \psi_{0}^{\prime}\left(\psi_{3}^{\prime}+\frac{1}{3} \bar{\psi}_{3}^{\prime}\right)-\psi_{0}^{\prime \prime}\left(\psi_{3}+\frac{1}{3} \bar{\psi}_{3}\right)=6\left(\theta_{0}+\psi_{0}^{\prime \prime \prime}\right), \\
& \psi_{0}^{\prime} \bar{\psi}_{3}-\bar{\psi}_{3} \psi_{0}^{\prime \prime}=0,
\end{aligned}
$$

$$
\begin{aligned}
& \psi_{0}^{\prime} \theta_{2}-\psi_{2} \theta_{0}^{\prime}=0, \\
& \psi_{0}^{\prime}\left(\theta_{3}+\frac{1}{3} \theta_{3}\right)-\left(\psi_{3}+\frac{1}{3} \bar{\psi}_{3}\right) \theta_{0}^{\prime}=6 \theta_{0}^{\prime \prime} / \sigma, \\
& \psi_{0}^{\prime} \theta_{3}-\bar{\psi}_{3} \theta_{0}^{\prime}=0 .
\end{aligned}
$$

The first equation in (5.9) may be integrated directly to give

$$
\psi_{2}=k \psi_{0}^{\prime}
$$

where $k$ is a constant determined by comparing the series for $\psi_{2}$ and $\psi_{0}^{\prime}$ in (5.7). Hence

$$
\psi_{2}=\frac{2 \alpha B_{1}}{3 a_{1}} \psi_{0}^{\prime}
$$

Similarly,

$$
\bar{\psi}_{3}=-\frac{2 \alpha C_{2}^{\prime}}{a_{1}} \psi_{0}^{\prime},
$$

and making use of the last two equations, the first and third in (5.10) give

$$
\theta_{2}=\frac{2 \alpha B_{1}}{3 a_{1}} \theta_{0}^{\prime}
$$

and

$$
\bar{\theta}_{3}=-\frac{2 \alpha C_{2}^{\prime}}{x_{1}} \theta_{0}^{\prime}
$$


Also, since $\bar{\psi}_{3}$ is a multiple of $\psi_{0}^{\prime}$, the equation for $\psi_{3}$ can be solved to give

$$
\psi_{3}=6 \psi_{0}^{\prime} \int^{y} \frac{\theta_{0}+\psi_{0}^{\prime \prime \prime}}{\psi_{0}^{\prime 2}} d y
$$

The integrand can be expanded in powers of $y$ using the series for $\theta_{0}$ and $\psi_{0}^{\prime}$. Hence

$$
\begin{aligned}
\psi_{3}= & -6\left(\frac{b_{0}+2 a_{2}}{a_{1}}\right)+y\left(m a_{1}-\frac{6 a_{2}\left(b_{0}+2 a_{2}\right)}{a_{1}}\right) \\
& +y \log y\left(\frac{6\left(b_{1}+6 a_{3}\right)}{a_{1}}-\frac{12 a_{2}\left(b_{0}+2 a_{2}\right)}{a_{1}^{2}}\right)+O\left(y^{2}\right),
\end{aligned}
$$

where $m$ is a constant of integration determined by comparison of (4.17) with the series for $\psi_{3}$ in (5.7). Thus

$$
m=\left\{\alpha\left(C_{2}+C_{2}^{\prime} \log \left(\frac{1}{3} \alpha\right)\right) / 3+a_{2}\left(b_{0}+2 a_{2}\right)\right\} / a_{1} .
$$

The equation for $\theta_{3}$ is simplified by using equation (5.15) to give

$$
\theta_{3}=\left(6 \theta_{0}^{\prime \prime} / \sigma+\psi_{3} \theta_{0}^{\prime}\right) / \psi_{0}^{\prime} \text {. }
$$

At this stage, it is interesting to note that not all the coefficients in the asymptotic series for the functions $f_{r}$ and $g_{r}$ are unknown; indeed by comparing the solutions obtained for $\psi_{2}, \psi_{3}, \psi_{3}^{\prime}$, with the series (5.7), we see that only one coefficient in the asymptotic series for each $f_{r}$ is unknown when those in its predecessors are known; this being the coefficient of $z$. The reason is clear; since one of the complementary functions (i.e. $\left.f_{r 2}\right)$ is a multiple of $z$, multiples of this function occur when finding a particular integral to the equation for $f_{r}$ and the appropriate factor has to be deter mined in each case. Moreover, when this has been done for each $f_{r}$, the asymptotic series for the functions $g_{r}$ are fully determined. In particular, we find that

$$
C_{2}^{\prime}=3\left(a_{1}\left(b_{1}+6 a_{3}\right)-2 a_{2}\left(b_{0}+2 a_{2}\right)\right) /\left(\alpha a_{1}^{2}\right),
$$

and from equations (3.2) we note that the terms containing multiples of $\log \xi$ (that is, the functions $\bar{\psi}_{3}$ and $\vec{\theta}_{3}$ ) vanish identically if the first two relations in (3.2) are satisfied. Further, the fact that some of the coefficients $A_{i}, B_{i}, C_{i}, \alpha_{i}, \beta_{i}$, etc., are known in terms of the coefficients in the initial profiles provides a useful check on the numerical computations.

\section{Uniformly heated plate with a temperature discontinuity}

The general theory developed in $\S 4$ and $\S 5$ is now applied to the flow induced by a vertical heated plate when there is an abrupt change of temperature from $T_{1}$ to $T_{2}\left(T_{1}, T_{2}\right.$ constants $)$ at a distance $L$ from the leading edge. Then $L$ provides a typical length scale for the problem. 

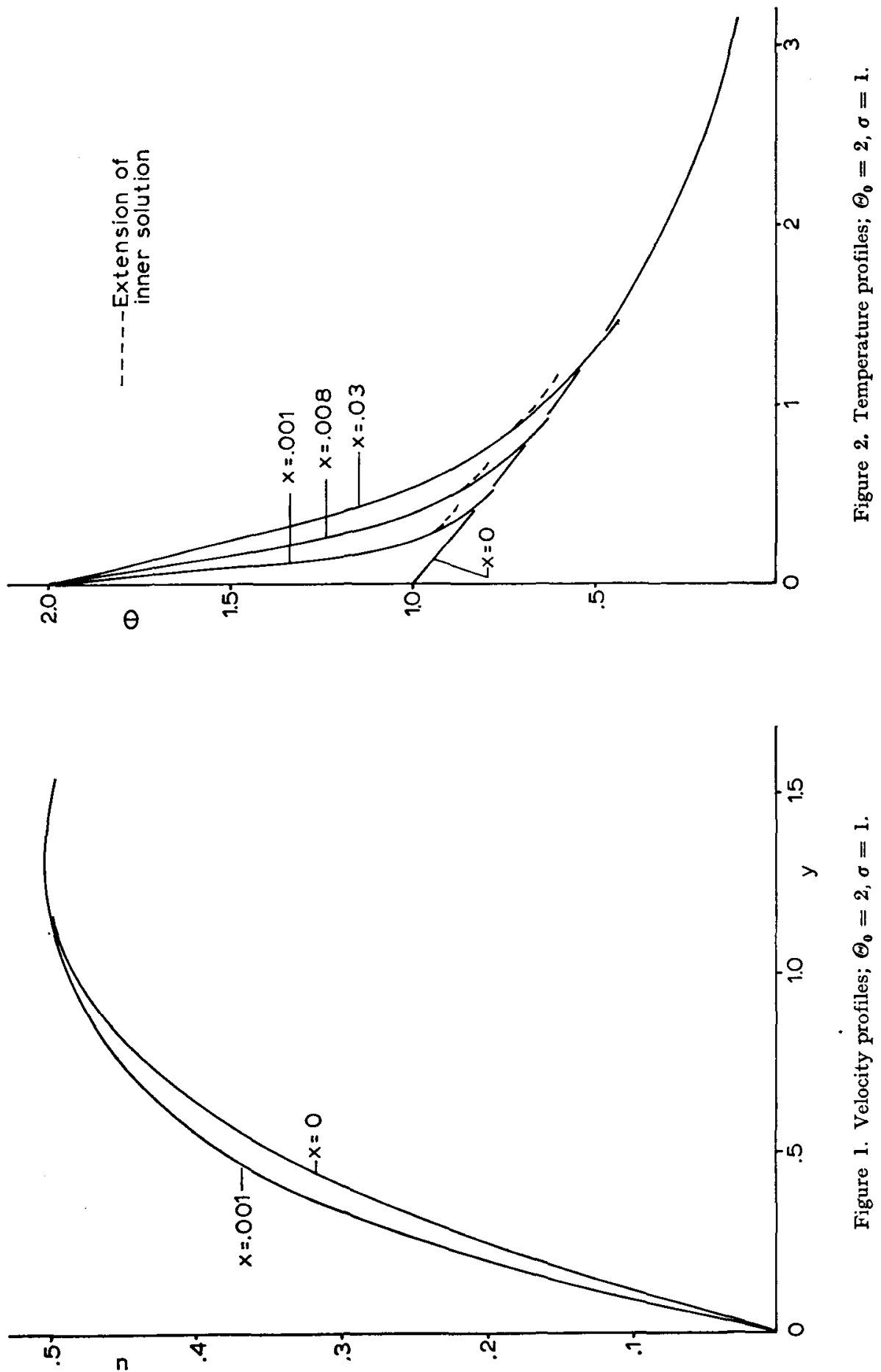

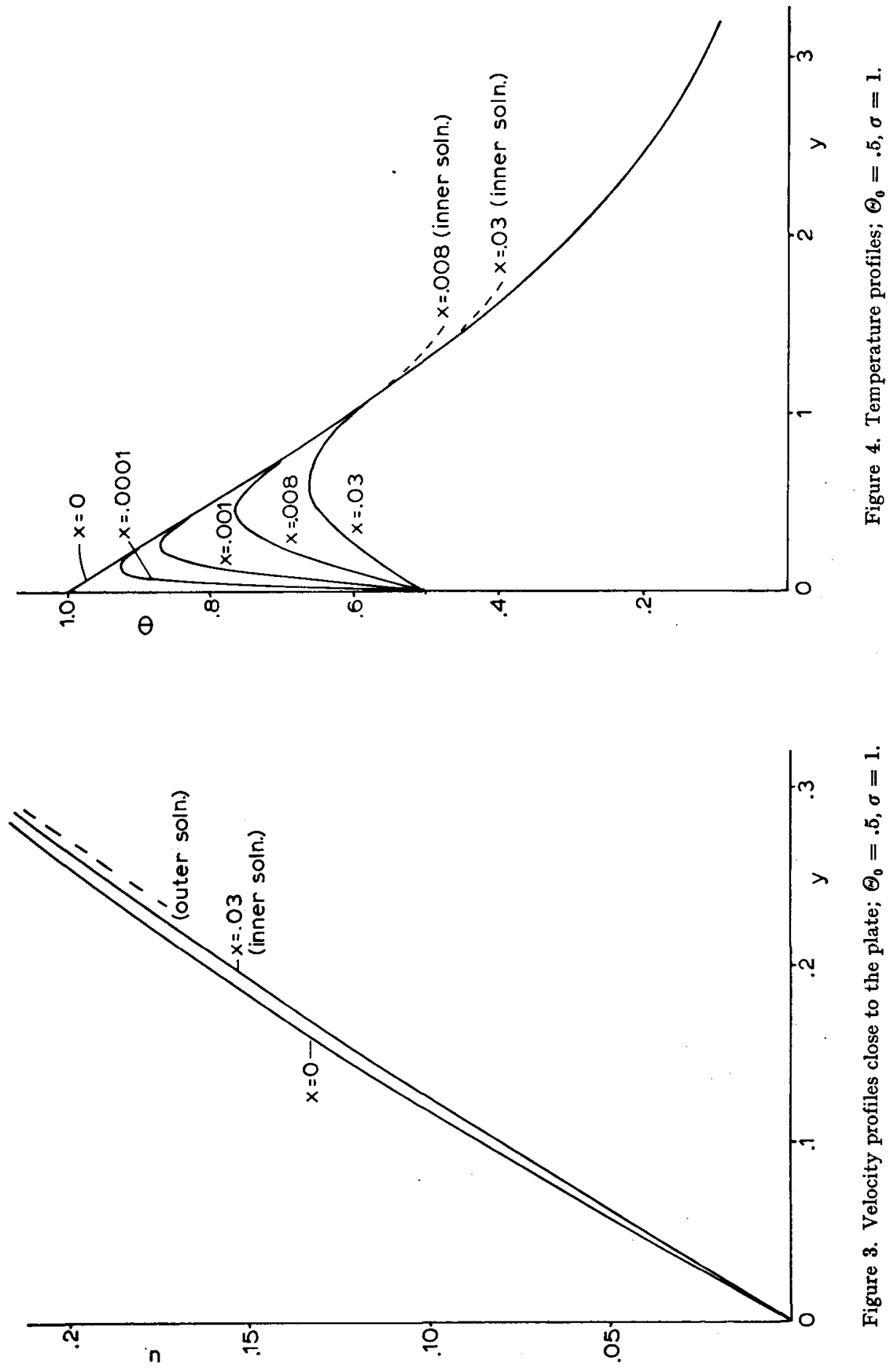
Moreover, if the origin of co-ordinates is taken at the discontinuity, then the initial profiles are given by the solution to the free convection problem outlined in $\S 2$, with $x$ (non-dimensionalized with respect to $L$ ) taken equal to unity (then $\zeta=y$ ). With starting values $F^{\prime \prime}(0)=p, G^{\prime}(0)=q$, the initial profiles are obtained by differentiating equations (2.8) and (2.9) and substituting in the Taylor series for $F$ and $G$ at the origin, using the boundary conditions (2.4) on $y=\mathbf{0}$. Hence

$$
\psi_{0}^{\prime}=p y-\frac{1}{2} y^{2}-\frac{1}{6} q y^{3}+\frac{1}{9} p^{2} y^{4}+O\left(y^{6}\right),
$$

and

$$
\theta_{0}=1+q y-\frac{\sigma p q}{32} y^{4}+O\left(y^{5}\right)
$$

Starting values, $p\left(=F^{\prime \prime}(0)\right)$ and $q\left(=G^{\prime}(0)\right)$ for $\sigma=1$ are given in $\S 2$.

Solutions are obtained for the two cases in which the temperature difference between the plate and the environment is suddenly doubled $\left(\Theta_{0}=2\right)$, or halved $\left(\Theta_{0}=0.5\right)$. Profiles for $u$ and $\theta$ at various downstream positions from the singularity are shown in Figs (1)-(4) for $\sigma=1$. These illustrate clearly the growth of the inner layer and in particular, the rapid erosion of the initial temperature profile to match the new wall condition. It is interesting to note that when the temperature difference is halved, the fluid near the wall is decelerated since this is now negatively buoyant with respect to adjacent fluid in the outer layer.

\section{Discussion}

The presence of a singularity (as defined in $\S 3$ ) at a particular station. along the plate results in the growth of an inner boundary layer from that point. As this layer spreads downstream, the original (outer) boundary layer is modified to accommodate it.

The expansions for the stream function and temperature in the inner layer (see $\S 4$ ) satisfy the equations of motion and the boundary conditions on the plate, but not those as $y \rightarrow \infty$. Furthermore, these expansions also depend on the coefficient $a_{i}, b_{i}$ and $\Theta_{i}$ and hence contain details of any singularity ${ }^{5}$ at $x=y=0$ (note, for example, that $g_{1}$ involved the difference $b_{0}-\Theta_{0}$ and therefore describes the immediate effect on the oncoming temperature field of a temperature discontinuity on the plate at $x=y=0$ ). The inner expansions are expected to be valid close to the plate, i.e. for $\eta=y x^{-\frac{1}{3}} \ll 1$ and for a range of $\xi=x^{\frac{1}{3}}<\xi_{0}$, say, where $\xi_{0} \ll 1$.

- Note - the expansion method is applicable whether or not a singularity is present but the power of the technique is most evident in the former case. 
The outer expansions describe the modifications of the oncoming flow due to the presence of the inner layer. They satisfy the equations of motion and the boundary conditions on $x=0$ and as $y \rightarrow \infty$ for $x \geq 0$. The forms of the expansions are obtained by the requirement that for small $y$, they are the same as the forms of the corresponding inner expansions for large $\eta$. Moreover, the terms in the outer expansions are determined uniquely by matching with the inner expansions as in $\S 5$. The outer expansions are expected to be valid for a range of $\xi$ similar to that of the inner expansions and for all but a small range of $y$ in the neighbourhood of the plate.

Immediately downstream of the origin $(x=y=0)$, one hopes to find a range of $\xi\left(<\xi_{1}\right.$ say), at each point of which there is a range of $y$ (depending on $\xi$ ) in which the inner and outer expansions for $\psi$ and $\theta$ overlap. For values of $\xi$ slightly larger than $\xi_{1}$, it may be possible to obtain the flow profiles by interpolation between the inner and outer solutions, but as $\xi \rightarrow 1$, none of the expansions is likely to converge. Unfortunately, these series are all too complicated to allow any convergence criteria to be worked out and it is necessary to rely on an appraisal of the solutions obtained in each case. It seems reasonable to believe that the solutions obtained here are convergent at least over the range of $x$ for which the inner and outer expansions overlap. In general, this region is that in which both the asymptotic forms of the inner solution and the series expansion about $y=0$ of the outer solucion give the same values for $\psi$ and $\theta$ for some range of $y$.

In the above discussion, it has been tacitly assumed that the Prandtl number of the fluid is of order unity. If the Prandtl number is either large or small compared to one, the thermal and momentum layers thicken at widely different rates and the inner expansions (and outer expansions) for each layer would be valid in different regions in the plane. It is not then clear how one might represent the coupling term (i.e. the buoyancy term) in the inner and outer solutions. As far as the author is aware, the case of extreme Prandtl number remains unsolved.

The continuation method described in this paper could be used in principle to advance the boundary layer solution indefinitely, in a step-bystep fashion. However, the convergence of the inner and outer expansions appears to be too slow for this to be practical and with high speed computing facilities available, a direct numerical solution of the equations seems a more preferable means of continuation. A finite difference procedure has been developed to this end, for free convection boundary layers, by Merkin [3]. The present theory is primarily intended to overcome the difficulties encountered at a singularity, where numerical methods break down. For this purpose the method of matched co-ordinate expansions is a powerful one, as is clearly illustrated by the solutions to the above problems. Moreove1, the expansions for $\psi$, and $\theta$ obtained here may be used to provide 
initial conditions for a numerical solution, once the singularity has been transversed.

\section{Acknowledgement}

The author wishes to express his gratitude to Mr. E. J. Watson for suggesting the problem and for a number of useful discussions.

\section{References}

[1] A. Erdelyi, Asymptotic Expansions, Dover, (1956), p. 21.

[2] S. Goldstein, Concerning some solutions of the boundary layer equations in hydrodynamics, Proc. Camb. Phil. Soc. 26 (1930), 1-30.

[3] J. Merkin, Ph. D. Thesis (Manchester University). (1968).

[4] S. Ostrach, An analysis of laminar free convection flow and heat transfer/about a flat plate parallel to the direction of the generating body force, NACA Rep. No. 1111, (1953), 17 pp.

[5] S. Ostrach, Laminar flows with body forces, Section F of Theory of Laminar Flows, (Ed. F. K. Moore), O.U.P., (1964).

[6] W. Rheinboldt, Zur Berechnung Stationärer Grenzschichten bei kontinuierlicher Absaugung mit unstetig veränderlicher Absaugegeschwindigkeit, J. Rat. Mech. Anal., 5 (1956), 539-604.

[7] L. J. Slater, The Confluent Hypergeometric Function, C.U.P., (1960), p. 59.

[8] E. J. Watson, The Continuation problem for boundary layer flow with suction, (1967), unpublished manuscript).

The University of Manchester

Manchester, U.K. 